\title{
Acquired Generalized Lipodystrophy
}

National Cancer Institute

\section{Source}

National Cancer Institute. Acquired Generalized Lipodystrophy. NCI Thesaurus. Code C131089.

Generalized lipodystrophy, the cause of which is not present at birth. 